\title{
Implant Therapy of Non-Syndromic Severe Oligodontia: A Clinical Report
}

\author{
Luis Gabriel Ladino, $D D S^{1^{*}}$ and Diego Rosselli, MD, EdM, MSc
}

${ }^{1}$ Private Practice in Implant Dentistry and Prosthodontics, Clinical Epidemiology and Biostatistics Master's Degree Program, Medical School, Pontificia Universidad Javeriana, Bogota, Colombia

${ }^{2}$ Associate Professor, Clinical Epidemiology and Biostatistics Department, Medical School, Pontificia Universidad Javeriana, Bogota, Colombia

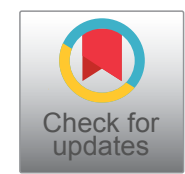

*Corresponding author: Luis Gabriel Ladino, Private Practice in Implant Dentistry and Prosthodontics, Clinical Epidemiology and Biostatistics Master's Degree Program, Medical School, Pontificia Universidad Javeriana, Av 9 No 127b-16. Office 401, Bogotá, Colombia, Tel: (+57)-3057979215

\section{Introduction}

In general, the term oligodontia refers to the failure to form six or more teeth, excluding the third molars, and may be associated with a mutation in the gen MSX1 and PAX9 [1,2]. Oligodontia also includes growth disturbances of the maxillofacial skeleton, tooth size variations and compromises facial appearance $[3,4]$. Treatment is often multidisciplinary, including different types of prostheses to improve both function and esthetics [4-6].

Agenesis of permanent teeth is the most common developmental dental abnormality. The prevalence of agenesis varies from $0.08 \%$ to $1.1 \%$, excluding third molars [7-9], and occurs mostly in second premolars and maxillary lateral incisors [10]. The absence of permanent teeth can cause vertical bone growth. In such instances, insufficient bone volume of the alveolar ridges can diminish the success rate of dental implants. Several augmentation techniques have been described to gain sufficient bone volume to support osseointegrated dental implants $[9,11]$. Augmentation of the maxillary sinus floor with different grafting material procedures has been used to gain sufficient bone volume for dental implants. Current evidence shows that maxillary sinus augmentation is a successful preprosthetic technique for augmentation of the edentulous posterior maxilla [12].
A prosthodontic treatment, including surgical approach, with bilateral sinus floor augmentation and simultaneous implant placement, in a patient with oligodontia and severe residual ridge reduction in the posterior region of the maxilla and mandible, is described in this clinical report.

\section{Case Description}

A healthy, 23-year-old, nonsmoking, partially edentulous woman was referred to prosthodontist private practice in Bogota, Colombia, with the complaint of malocclusion, and an unattractive smile resulting from absence of some teeth. She had received fixed orthodontic treatment. She never used prostheses and refused using removable partial denture. Clinical, intraoral, and radiographic examinations were performed, and diagnostic casts were made (Figure 1a, Figure $1 \mathrm{~b}$, Figure $1 \mathrm{c}$ and Figure $1 \mathrm{~d}$ ).

Intraoral status revealed absence of all lateral incisors and second premolars; in addition, absence of mandibular second molars that never were formed. Second temporary right maxillary molar and second temporary left mandibular molar were present, without radiographic evidence of a root. Canines, both maxillary and mandibular, had been moved to act as lateral incisors (Figure 1d). In addition, a reduction in occlusal vertical dimension produced esthetic facial problems. Intraoral examination also revealed inadequate bilateral 


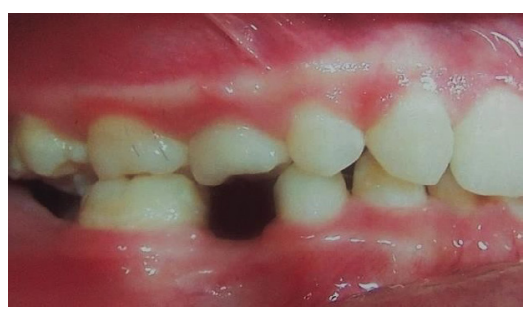

A

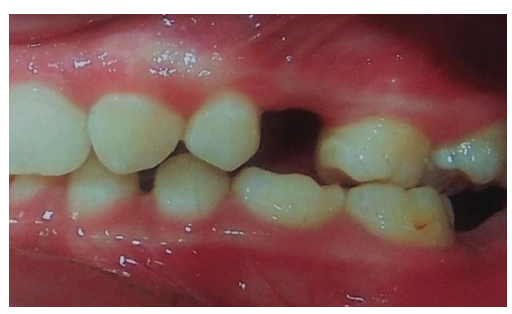

B

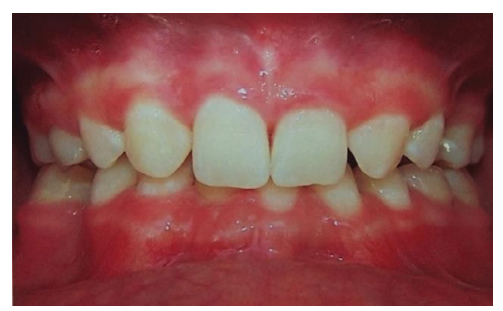

C

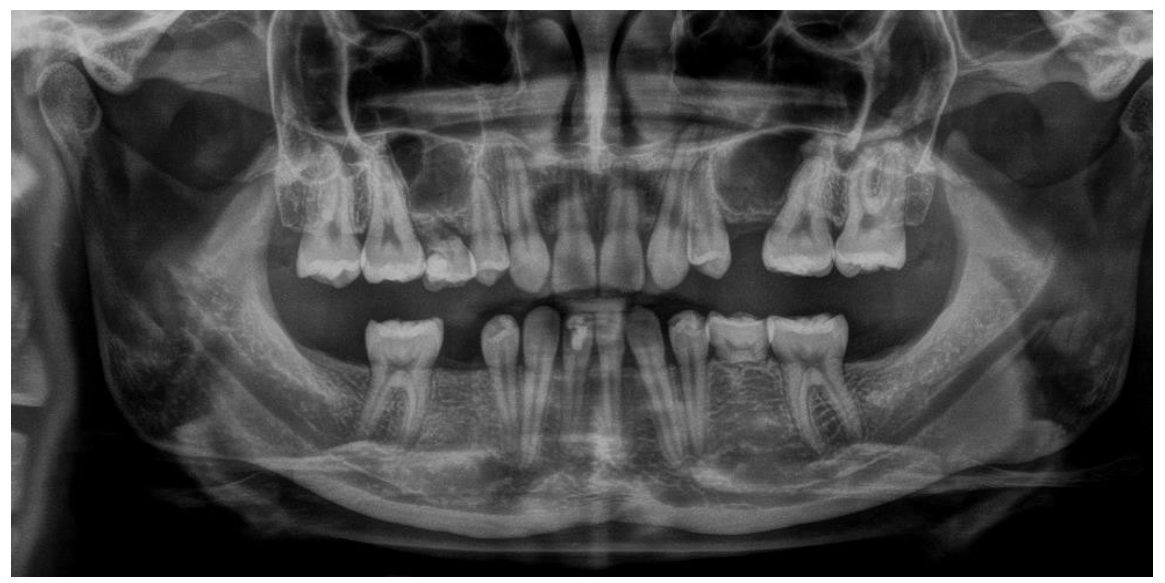

D

Figure 1: Frontal and Lateral occlusal view showing initial situation (a) Right maxillary region; (b) Left maxillary region; (c) Anterior region and (d) Baseline Panoramic radiographic.

posterior maxillary alveolar bone development, due to the neumatization of maxillary sinus secondary to lack of formation of permanent teeth.

The patient was informed about the consequences of missing teeth, and about treatment options. A diagnosis of nonsyndromic oligodontia was proposed. However, the family history was positive for oligodontia; the patient's aunt, and a female cousin presented absence of some permanent teeth and had delayed eruption of the permanent teeth. The patient was offered implant-supported crowns; she consented to treatment involving several surgical procedures.

\section{Surgical procedures}

Informed patient consent was obtained prior to treatment. All surgical operations were performed under sedation and local anesthesia.

Bilateral sinus lifting procedures were made first. Both right and left residual bone heights were 2 to 3 $\mathrm{mm}$, with at least $5 \mathrm{~mm}$ bone width below the maxillary sinus. Patient received a bilateral 1-stage lateral window sinus lift with simultaneous implant placement including a bone graft. This procedure was made using a non-restrictive handmade guide to improve accuracy of window locations, and implants placement. This guide design was made based on periapical radiographs and CT scan, and allowed a better control of window size and implant position. Using this guide, a window osteotomy of lateral alveolar bone was made (Figure 2a, Figure $2 b$, Figure $2 c$ and Figure $2 d$ ); the sinus membrane was then lifted, and the mobilized portion of the sinus wall removed. The space created after sinus membrane elevation was grafted with BioOss Geitslich $^{\circledR}$ and the lateral sinus wall defect was covered using a resorbable collagenous membrane. The mucoperiosteal flap was then sutured. Antibiotic therapy with amoxycilin $500 \mathrm{mg}$ tid was prescribed for 7 days; a nonsteroidal analgesic was also recommended.

Postsurgical instructions included a soft diet for 2 weeks and appropriate oral hygiene control twice daily with chlorhexidine mouthwash.

In addition to simultaneous implant placement with bilateral sinus floor augmentation in the maxilla, four dental implants were placed in the mandible $(4.1 \times 10$ $\mathrm{mm}[\mathrm{n}=3] ; 4.1 \times 10 \mathrm{~mm}[\mathrm{n}=4] ;$ and $3.3 \times 12 \mathrm{~mm}[\mathrm{n}$ $=1 \mathrm{]}$; $3 \mathrm{i}$ Biomed). At the time of abutment connection, implant integration was checked both clinically and radiographically.

The patient was recalled every 2 months for 6 months for clinical and radiologic evaluation. The success criteria included: Immobility of the implant, absence of pain or suppuration, radiographically visible direct implant-to-bone contact, and less than $1.0 \mathrm{~mm}$ vertical bone resorption in the first year after prosthetic loading.

\section{Prosthodontic rehabilitation}

After 6 months, full-arch impressions of the maxillary and mandibular arch were taken, then the cast models 

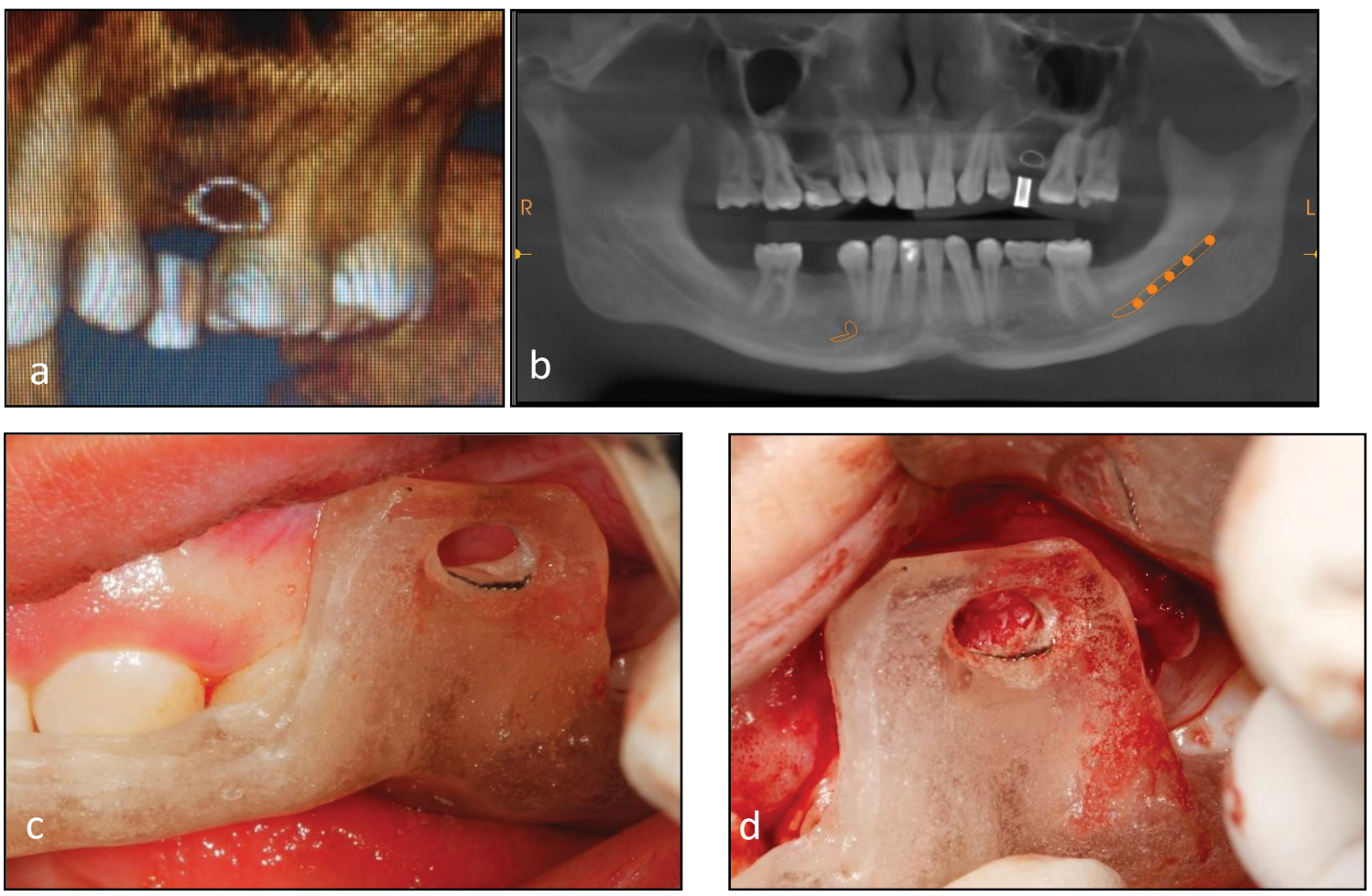

Figure 2: $(a, b)$ Tomographic guide; (c) Clinical non-restrictive handmade surgical guide to make the window for sinus lift and (d) Sinus grafted.

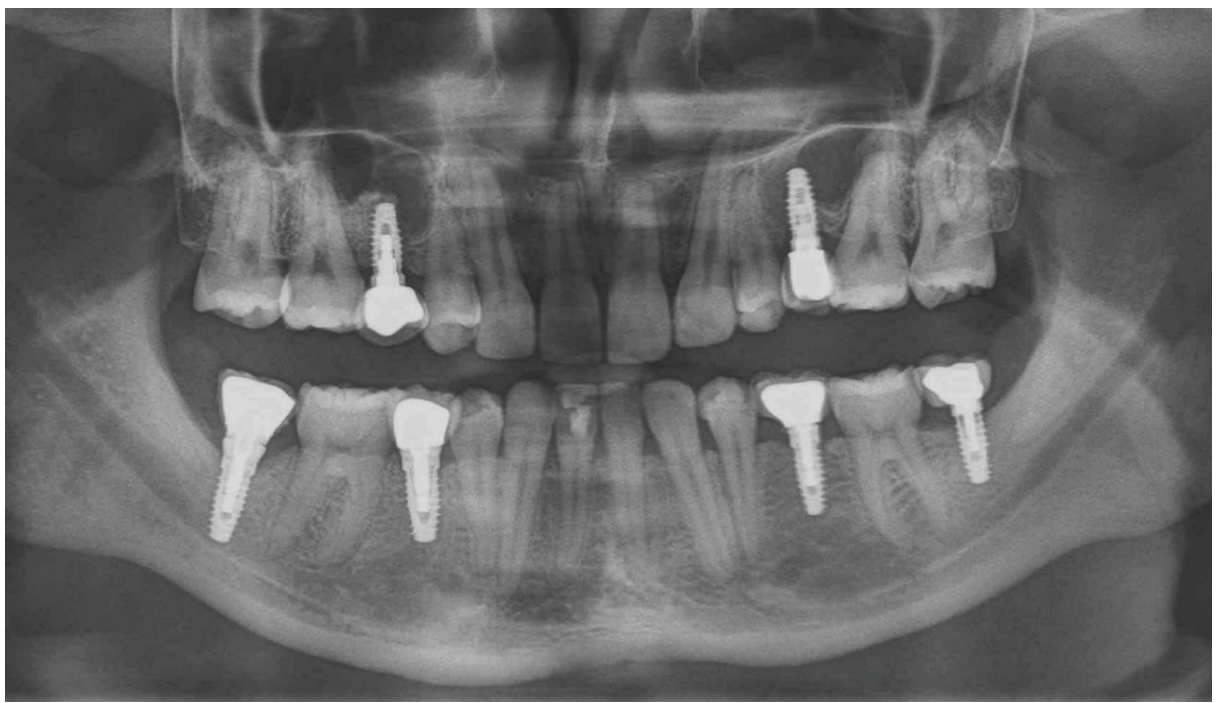

Figure 3: Final panoramic radiographic.

were mounted on a semi adjustable articulator. The intermaxillary relationship was evaluated and prosthetic occlusal plane was leveled through the metal-ceramic implant-supported restorations.

In this clinical report, definitive restorations were carried out using conventional cement- retained and screw-retained metal ceramic crowns to allow normal masticatory function and phonetic function as well. Radiographs were taken to evaluate the implantabutment restoration fit (Figure 3). The patient was recalled one week later to evaluated oral hygiene and 12 months later again without any complications. After prosthetic rehabilitation, the facial appearance and the occlusion were improved, In addition esthetic and psychological expectations of patient were carried out.

Oligodontia is a number anomaly and is the most common dental anomaly that can affect patients with syndrome or non-syndrome [13]; Some studies reported mutations in the genes that were associated with nonsyndromic oligodontia, mutation of gene MXS1 or PAX9 may create an impairment of one or more molecular processes that regulate tooth formation [1,14] and some studies report that it may be greater in women than in men [3]. 
The most frequently missing teeth reported are maxillary o mandibular second premolar and maxillary lateral incisors. In accordance with other studies, in this clinical report, the mandibular second premolars, maxillary and mandibular lateral incisors, and mandibular second molars [13]. In many cases the absence of several teeth can compromise aesthetics, function and psychologically [15].

Treatments to replace missing teeth include, removable partial dentures, fixed prostheses, and more recently implant-supported restorations $[16,17]$, these treatments may require advanced surgical procedures to gain bone volume, among which the most commonly used is to augmentation sinus lift for implant placement. Elevation of maxillary sinus simultaneous to implant placement using autologous bone and other bone substitutes it's a predictable treatment to dental implant survival, even sinus lifting with simultaneous implant placement could be used to treat atrophic maxilla in patients with minimal residual alveolar bone height when initial stability could be obtained by using taper designed implants [18].

Cases of severe oligodontia should be treated with a multidisciplinary team that may include periodontics, orthodontics, prosthodontics, and maxillofacial surgery; and their work must be oriented to reduce the rate of complications and carried out the expectations of patients.

\section{Conclusion}

This clinical report concerns a patient with severe non-syndromic oligodontia. The patient was treated with bilateral sinus lift surgery before implant therapy. In addition, she was rehabilitated with implant-supported crowns to recovery the masticatory and esthetics function. It is possible to achieve excellent results with implant therapy in cases of severe oligodontia recovering functional abilities and adequate esthetics. It's very important keep in mind a previous interdisciplinary evaluation and planning to get successful results.

\section{Acknowledgments}

The authors reported no conflicts of interest related to this study.

\section{References}

1. Swinnen S, Bailleul-Forestier I, Arte $S$, Nieminen $P$, Devriendt K, et al. (2008) Investigating the etiology of multiple tooth agenesis in three sisters with severe oligodontia. Orthod Craniofac Res 11: 24-31.

2. Kim JW, Simmer JP, Lin BPJ, Hu JCC (2006) Novel MSX1 frameshift causes autosomal-dominant oligodontia. J Dent Res 85: 267-271.

3. Créton MA, Cune MS, Verhoeven W, Meijer GJ (2007) Patterns of missing teeth in a population of oligodontia patients. Int J Prosthodont 20: 409-413.

4. Gunbay T, Koyuncu BO, Sipahi A, Bulut H, Dundar M
(2011) Multidisciplinary approach to a nonsyndromic oligodontia patient using advanced surgical techniques. Int J Periodontics Restorative Dent 31: 297-305.

5. Worsaae N, Jensen BN, Holm B, Holsko J (2007) Treatment of severe hypodontia-oligodontia--an interdisciplinary concept. Int J Oral Maxillofac Surg 36: 473-480.

6. Sclar AG, Kannikal J, Ferreira CF, Kaltman SI, Parker WB (2009) Treatment planning and surgical considerations in implant therapy for patients with agenesis, oligodontia, and ectodermal dysplasia: Review and case presentation. J Oral Maxillofac Surg 67: 2-12.

7. Khalaf K, Miskelly J, Voge E, Macfarlane TV (2014) Prevalence of hypodontia and associated factors: A systematic review and meta-analysis. J Orthod 41: 299316.

8. Bedoya RA, Collo QL, Gordillo MI, Yusti SA, Tamayo CJA, et al. (2014) Anomalías dentales en pacientes de ortodoncia de la ciudad de Cali, Colombia. Rev CES Odont 27: 45-54.

9. Filius MA, Cune MS, Raghoebar GM, Vissink A, Visser A (2016) Prosthetic treatment outcome in patients with severe hypodontia: A systematic review. J Oral Rehabil 43: 373-387.

10. Rakhshan V, Rakhshan H (2016) Meta-analysis and systematic review of the number of nonsyndromic congenitally missing permanent teeth per affected individual and its influencing factors. Eur J Orthod 38: 170-177.

11. Créton $M$, Cune $M$, Verhoeven $W$, Muradin $M$, Wismeijer D, et al. (2010) Implant treatment in patients with severe hypodontia: a retrospective evaluation. J Oral Maxillofac Surg 68: 530-538.

12. Danesh-Sani SA, Loomer PM, Wallace SS (2016) A comprehensive clinical review of maxillary sinus floor elevation: Anatomy, techniques, biomaterials and complications. Br J Oral Maxillofac Surg 54: 724-730.

13. Mahadevi BH, Puranik RS, Shrinivas SV (2011) Oligodontia: A case report and review of literature. World J Dent 2: 259262.

14. Kang HG, Huh YH, Park CJ, Cho LR (2016) Rehabilitation of a patient with non-syndromic partial oligodontia. J Adv Prosthodont 8: 241-250.

15. Zhang XX, Peng D, Feng HL (2015) Prosthodontic treatment for severe oligodontia with long-term follow-up. Chin J Dent Res 18: 163-169.

16. Durey K, Cook P, Chan M (2014) The management of severe hypodontia. Part 1: Considerations and conventional restorative options. Br Dent J 216: 25-29.

17. Giray B, Akca K (2003) Two-year follow-up of a patient with oligodontia treated with implant-and tooth-supported fixed partial dentures: A case report. Int J Oral Maxillofac Implants 18: 905-911.

18. Cha HS, Kim A, Nowzari H, Chang HS, Ahn KM (2014) Simultaneous sinus lift and implant installation: prospective study of consecutive two hundred seventeen sinus lift and four hundred sixty-two implants. Clin Implant Dent Relat Res 16: 337-347. 